\title{
Mitochondrial dysfunction rather than mtDNA sequence mutation is responsible for the multi-drug resistance of small cell lung cancer
}

\author{
LIJIE MA $^{1 *}$, RUIXUAN WANG ${ }^{1 *}$, HONGTAO DUAN ${ }^{3}$, YANDONG NAN $^{1}$, \\ QINGWEI WANG ${ }^{2}$ and FAGUANG JIN ${ }^{1}$ \\ Departments of ${ }^{1}$ Respiration, ${ }^{2}$ Pharmacy and ${ }^{3}$ Thoracic Surgery, Tangdu Hospital, \\ Fourth Military Medical University, Xi'an, Shaanxi 710038, P.R. China
}

Received April 29, 2015; Accepted September 9, 2015

DOI: $10.3892 /$ or.2015.4315

\begin{abstract}
Small cell lung cancer (SCLC) accounts for $\sim 15 \%$ of all lung cancer cases, and chemotherapy has dramatically improved the survival rate of SCLC patients. Yet, the long-term survival rate of this cancer has not improved since multi-drug resistance (MDR) may emerge after chemotherapy. Mitochondrial DNA (mtDNA) mutation-related biological processes, such as energy metabolism and reactive oxygen species (ROS) production, have been considered to be associated with tumorigenesis and drug resistance. It was hypothesized and demonstrated, in the present study, that mitochondrial dysfunction is the reason for the occurrence and progression of SCLC. mtDNA from drug sensitive and drug insensitive cell lines (H446 and H446/CDDP) was sequenced and compared with the revised Cambridge reference sequence (rCRS). The results revealed that there was no difference in the mtDNA sequence from H446 and H446/CDDP cells, but several spot mutations were observed according to that of rCRs. Further evaluation on mitochondrial function revealed that H446 cells synthesized and secreted more lactic acid and ROS compared with that of H446/CDDP cells when challenged by the same dose of cisplatin ( $\mathrm{P}>0.05)$. In addition, examination of the mitochondrial apoptotic pathway indicated that more Bax, cleaved caspase- 3 and cleaved caspase- 9 were expressed
\end{abstract}

Correspondence to: Professor Faguang Jin, Department of Respiration, Tangdu Hospital, Fourth Military Medical University, Xi'an, Shaanxi 710038, P.R. China

E-mail: jinfag@fmmu.edu.cn

Professor Qingwei Wang, Department of Pharmacy, Tangdu Hospital, Fourth Military Medical University, Xi'an, Shaanxi 710038, P.R. China

E-mail:wqwlxy@163.com

*Contributed equally

Key words: small cell lung cancer, mitochondrial dysfunction, multi-drug resistance, mitochondrial apoptotic pathway in H446 cells compared with that of H446/CDDP cells when stimulated by the same dose of cisplatin $(\mathrm{P}>0.05)$. In conclusion, the results of the present study revealed that mtDNA mutations were responsible for the tumorigenesis of SLCL, but not associated with the drug sensitivity of SCLC cell lines. On the other hand, varied mitochondrium content-related mitochondrial dysfunction participated in the MDR of SCLC possibly by affecting the ROS-mediated mitochondrial apoptotic pathway.

\section{Introduction}

Small cell lung cancer (SCLC) accounts for $\sim 15 \%$ of all lung cancer cases $(1,2)$; however, increased attention has been paid to this histotype due to its rapid growth and early dissemination to regional lymph nodes and remote organs (3). Chemotherapy has dramatically improved the survival rate of SCLC patients, yet multi-drug resistance (MDR), emerging after chemotherapy, leads to relapse and mortality (4). MDR has become the major clinical obstacle to the treatment of SCLC. New chemotherapeutics and adjustment of chemotherapy programs have not improved the long-term survival rates. Research on the mechanisms of MDR in SCLC has continuously increased since a main treatment for SCLC has not markedly evolved during the past three decades.

To date, it has not been fully elucidated how acquired MDR evolves, yet various meaningful theories and ideas have guided the direction of research efforts. Research has confirmed that MDR is caused by different mechanisms. What is more, there could be more than one mechanism involved in one type of cancer $(5,6)$. Moreover, a theory, known as the 'Warburg effect', concerning energy metabolism reprogramming was introduced and recognized to be closely related to tumorigenesis and cancer progression. Warburg first proposed that cancer cells, different from normal cells, increase their glycolytic activity and reduce mitochondrial respiration whether there is an abundant supply of oxygen or not $(7,8)$. Increasing evidence indicates that numerous molecular mechanisms are involved in the Warburg effect (9), and mitochondria were first to be indicated since this organelle has been so closely related to energy metabolism and cell survival. An abundance 
of research indicates that mitochondrial dysfunction resulting from mutation in mitochondrial DNA (mtDNA) is responsible for tumorigenesis (10).

Mitochondrial dysfunction is also believed to provide a survival benefit to cancer cells (11). It has been reported in several cancer types such as prostate (12), kidney (13), breast (14), liver (15), colon and rectal cancer cells that mutations and/or depletion of mtDNA are the reasons for the multi-drug-resistant features (16). More importantly, chemotherapy-induced low levels of mtDNA have been proven to be related to acquired drug resistance and anti-apoptotic properties of cancer cells $(17,18)$. A typical example was that mtDNA mutations and reduced mtDNA copy number in breast and prostate cancer patients were associated with enhanced metastasis and poor patient prognosis $(12,17,18)$. It appears that mitochondrial dysfunction resulting from mtDNA mutation is closely associated with cancer occurrence and progression.However, there is no research clearly illustrating the possible role of mtDNA mutation in tumorigenesis and MDR of SCLC.

Thus, we investigated the sequence of mtDNA from SCLC cell lines, H446 and H446/CDDP, and compared these sequences with the revised Cambridge reference sequence (rCRS) (19) in order to ascertain whether mtDNA mutations participate in the occurrence and acquired MDR of SCLC. After that, the differences in mitochondrial function were evaluated between H446 and H446/CDDP cells. Finally, we investigated the activation of the mitochondrial apoptotic pathway in H446 and H446/CDDP cells challenged by cisplatin in order to illustrate the possible mechanism of the mitochondrial dysfunction in the MDR of SCLC.

\section{Materials and methods}

Cell lines and culture. Small cell lung cancer cell line H446 and its multi-drug-resistant cell line H446/CDDP were kindly gifted by Dr Guisheng Qian (Institute of Human Respiratory Disease, Xinqiao Hospital, Third Military Medical University). The H446 cell line was purchased from the American Type Culture Collection (ATCC; Manassas, VA, USA) and the MDR properties of H446/CDDP cells were induced by a low dosage of cisplatin. These cells were maintained in RPMI1640 medium with $10 \%$ fetal calf serum (Gibco-BRL, Grand Island, NY, USA) in a humidified atmosphere containing $5 \% \mathrm{CO}_{2}$ at $37^{\circ} \mathrm{C}$. In order to maintain the MDR properties of the H446/CDDP cells, cisplatin (with a final concentration of $0.5 \mu \mathrm{g} / \mathrm{ml}$ ) was added to the culture medium for the H446/CDDP cells.

Evaluation of drug resistance. The sensitivity of $\mathrm{H} 446$ and H446/CDDP cells to cisplatin was evaluated by a microculture tetrazolium (MTT) assay. Briefly, the cells were seeded in 96-well plates at a density of $1 \times 10^{4}$ cells/well. The cells were treated with $5 \mu \mathrm{g} / \mathrm{ml}$ cisplatin for 24,48 and $72 \mathrm{~h}$ when grown to confluency. The supernatant was discarded at corresponding time-points, and $20 \mu \mathrm{l}$ MTT $(5 \mathrm{mg} / \mathrm{ml}$, dissolved in PBS and filtered through a $0.22-\mathrm{mm}$ membrane) was added into each well and incubated for $4 \mathrm{~h}$ at $37^{\circ} \mathrm{C}$. Finally, the absorption values were determined at $492 \mathrm{~nm}$ on an automated Bio-Rad 550 microtiter plate reader.
Extraction of genomic DNA. Genomic DNAs were extracted from the H446 and H446/CDDP cells in a logarithmic growth phase according to the genomic DNA extraction kit instructions (Tiangen Biotechnology Co., Beijing, China). Optical density values of A260 and A280 were determined by using an ultraviolet spectrophotometer in order to evaluate the purity of the DNAs.

Amplification and sequencing of mtDNA. mtDNAs were amplified by using 26 primers (sequences shown in Table I) according to previous research (12). To amplify mtDNA in the genomic DNA samples, $1 \mu \mathrm{g}$ of diluted genomic DNA was mixed with paired primers (primers were provided by AuGCT Biotechnology Co., Beijing, China). Along with genomic sequences, Premix Taq version 2.0 (Takara Biotechnology Co., Dalian, China) was added into a $20-\mu 1$ total volume. The mtDNA was amplified under the following conditions: 35 cycles, and the cycling conditions were as follows: $95^{\circ} \mathrm{C}$ for $30 \mathrm{sec} ; 54^{\circ} \mathrm{C}$ for $30 \mathrm{sec}$; and $72^{\circ} \mathrm{C}$ for $1 \mathrm{~min}$. After that, the PCR products were purified and sequenced by ABI Prism 3700 sequencing machine.

Mitochondrial ultrastructure examination. Electron microscopy was performed on H446 and H446/CDDP cells in order to investigate whether the difference of mitochondrial structure was related to the varied sensitivity of H446 and H446/CDDP cells to cisplatin. Cell mass was collected by centrifugal method, and $1 \mu \mathrm{m}$ thick sections were stained to locate significant areas for electron microscopic examination. Ultrathin sections were stained with uranyl acetate, and mitochondria were examined under a TEM-100CX electron microscope (Japan Electron Optics Laboratory, Tokyo, Japan) after the cell mass was post-fixed in osmium tetroxide for $1 \mathrm{~h}$, dehydrated in alcohol, and embedded in epoxy resin.

Mitochondrial staining. MitoTracker probe (Life Technologies Corporation, USA) was used to dye mitochondria in the H446 and H446/CDDP cells, and the procedure was carried out according to the manufacturer's instructions. Briefly, medium was removed from the H446 and H446/CDDP cells in logarithmic growth phase, and the cells were washed 3 times with phosphate-buffered saline (PBS; Life Technologies Corporation). The cells were then incubated with warmed $\left(37^{\circ} \mathrm{C}\right)$ staining solution containing MitoTracker probe $(100 \mathrm{nM})$ for $30 \mathrm{~min}$. After that, the cells were washed again with PBS and examined with an inverted fluorescence microscope (DMI6000B; Leica, Germany) within half an hour.

Detection of lactic acid secretion. In order to determine the metabolic difference of H446 and H446/CDDP cells, the lactic acid content in the cultural medium with or without $5 \mu \mathrm{g} / \mathrm{ml}$ cisplatin stimulation was detected with a lactic acid assay kit (Jiancheng Bioengineering Institute, Nanjing, China) at different time-points. The procedure was carried out according to the manufacturer's instructions, and the data were collected and are expressed as mean \pm standard deviation.

Reactive oxygen species (ROS) detection. In order to evaluate the function of mitochondria in the H446 and H446/CDDP cells, ROS levels in the cells with or without cisplatin stimu- 
Table I. Sequences of primers for the mtDNAs used in the present research.

\begin{tabular}{|c|c|c|c|c|c|}
\hline Primer pair no. & Primer no. & Sequence $5^{\prime}-3^{\prime}$ & $\begin{array}{l}3 \text { ' combined } \\
\text { location }\end{array}$ & Length (bp) & $\begin{array}{l}\text { Overlapping length of } \\
\text { two-way sequencing (bp) }\end{array}$ \\
\hline \multirow[t]{2}{*}{1} & $1 \mathrm{~F}$ & CTCCTCAAAGCAATACACTG & 611 & 840 & 202 \\
\hline & $1 \mathrm{R}$ & TGCTAAATCCACCTTCGACC & 1411 & & \\
\hline \multirow[t]{2}{*}{2} & $2 \mathrm{~F}$ & CGATCAACCTCACCACCTCT & 1245 & 802 & 204 \\
\hline & $2 \mathrm{R}$ & TGGACAACCAGCTATCACCA & 2007 & & \\
\hline \multirow[t]{2}{*}{3} & $3 \mathrm{~F}$ & GGACTAАCСССТАТАССТTCTGC & 1854 & 860 & 196 \\
\hline & $3 R$ & GGCAGGTCAATTTCACTGGT & 2669 & & \\
\hline \multirow[t]{2}{*}{4} & $4 \mathrm{~F}$ & AAATCTTACCCCGCCTGTTT & 2499 & 887 & 208 \\
\hline & $4 \mathrm{R}$ & AGGAATGCCATTGCGATTAG & 3346 & & \\
\hline \multirow[t]{2}{*}{5} & $5 \mathrm{~F}$ & TACTTCACAAAGCGCCTTCC & 3169 & 832 & 215 \\
\hline & $5 \mathrm{R}$ & ATGAAGAATAGGGCGAAGGG & 3961 & & \\
\hline \multirow[t]{2}{*}{6} & $6 \mathrm{~F}$ & TGGCTCCTTTAACСТCTCCA & 3796 & 898 & 203 \\
\hline & $6 \mathrm{R}$ & AAGGATTATGGATGCGGTTG & 4854 & & \\
\hline \multirow[t]{2}{*}{7} & $7 \mathrm{~F}$ & ACTAATTAATCCCCTGGCCC & 4485 & 975 & 207 \\
\hline & $7 \mathrm{R}$ & CCTGGGGTGGGTTTTGTATG & 5420 & & \\
\hline \multirow[t]{2}{*}{8} & $8 \mathrm{~F}$ & CTAACCGGCTTTTTGCCC & 5255 & 814 & 201 \\
\hline & $8 \mathrm{R}$ & ACCTAGAAGGTTGCCTGGCT & 6031 & & \\
\hline \multirow[t]{2}{*}{9} & $9 \mathrm{~F}$ & GAGGCCTAACCCCTGTCTTT & 5855 & 827 & 214 \\
\hline & $9 \mathrm{R}$ & ATTCCGAAGCCTGGTAGGAT & 6642 & & \\
\hline \multirow[t]{2}{*}{10} & $10 \mathrm{~F}$ & CTCTTCGTCTGATCCGTCCT & 6469 & 886 & 211 \\
\hline & $10 \mathrm{R}$ & AGCGAAGGCTTCTCAAATCA & 7315 & & \\
\hline \multirow[t]{2}{*}{11} & $11 \mathrm{~F}$ & ACGCCAAAATCCATTTCACT & 7148 & 987 & 205 \\
\hline & $11 \mathrm{~F}$ & CGGGAATTGCATCTGTTTTT & 8095 & & \\
\hline \multirow[t]{2}{*}{12} & $12 \mathrm{~F}$ & ACGAGTACACCGACTACGGC & 7937 & 900 & 196 \\
\hline & $12 \mathrm{R}$ & TGGGTGGTTGGTGTAAATGA & 8797 & & \\
\hline \multirow[t]{2}{*}{13} & $13 \mathrm{~F}$ & TTTCССССТCTATTGATCCC & 8621 & 816 & 214 \\
\hline & $13 \mathrm{R}$ & GTGGCCTTGGTATGTGCTTT & 9397 & & \\
\hline \multirow[t]{2}{*}{14} & $14 \mathrm{~F}$ & CCСACCAATCACATGCCTAT & 9230 & 940 & 205 \\
\hline & $14 \mathrm{R}$ & TGTAGCCGTTGAGTTGTGGT & 10130 & & \\
\hline \multirow[t]{2}{*}{$15-1$} & $15-1 \mathrm{~F}$ & CTTCTATTGATGAGGGTCTT & 9991 & 670 & 218 \\
\hline & $15-1 \mathrm{R}$ & GGTGTTGAGGGTTATGAGA & 10622 & & \\
\hline \multirow[t]{2}{*}{$15-2$} & $15-2 \mathrm{~F}$ & AAGGATTAGACTGAACCGAA & 10404 & 627 & 135 \\
\hline & $15-2 \mathrm{R}$ & CTGATTGTGAGGGGTAGGA & 10992 & & \\
\hline \multirow[t]{2}{*}{$15-3$} & $15-3 \mathrm{~F}$ & CAACCACCCACAGCCTAA & 10857 & 693 & 198 \\
\hline & $15-3 R$ & TTGAGAATGAGTGTGAGGCG & 11512 & & \\
\hline \multirow[t]{2}{*}{17} & $17 \mathrm{~F}$ & TCACTCTCACTGCCCAAGAA & 11314 & 802 & 196 \\
\hline & $17 \mathrm{R}$ & GGAGAATGGGGGATAGGTGT & 12076 & & \\
\hline \multirow[t]{2}{*}{18} & $18 \mathrm{~F}$ & ТАТСАСТСТССТАСТТАСАG & 11948 & 866 & 166 \\
\hline & $18 \mathrm{R}$ & AGAAGGTTATAATTCCTACG & 12772 & & \\
\hline \multirow[t]{2}{*}{19} & $19 \mathrm{~F}$ & AAACAACCCAGCTCTCCCTAA & 12571 & 977 & 242 \\
\hline & $19 \mathrm{R}$ & TCGATGATGTGGTCTTTGGA & 13507 & & \\
\hline 20 & $20 \mathrm{~F}$ & ACATCTGTACCCACGCCTTC & 13338 & 970 & 207 \\
\hline & $20 \mathrm{R}$ & AGAGGGGTCAGGGTTCATTC & 14268 & & \\
\hline 21 & $21 \mathrm{~F}$ & GCATAATTAAACTTTACTTC & 14000 & 938 & 206 \\
\hline & $21 \mathrm{R}$ & AGAATATTGAGGCGCCATTG & 14998 & & \\
\hline 22 & $22 \mathrm{~F}$ & TGAAACTTCGGCTCACTCCT & 14856 & 1,162 & 180 \\
\hline & $22 \mathrm{R}$ & AGCTTTGGGTGCTAATGGTG & 15978 & & \\
\hline 23 & $23 \mathrm{~F}$ & TCATTGGACAAGTAGCATCC & 15811 & 765 & 190 \\
\hline & $23 \mathrm{R}$ & GAGTGGTTAATAGGGTGATAG & 5 & & \\
\hline 24-1 & $24-1 \mathrm{~F}$ & CATTATCCCGCACAAGAGTG & 16419 & 420 & 160 \\
\hline & $24-1 \mathrm{R}$ & TGGAAAGTGGCTGTGCAGACAT & 250 & & \\
\hline $24-2$ & $24-2 \mathrm{~F}$ & CTTTGATTCCTGCCTCATCC & 132 & 540 & 100 \\
\hline & $24-2 R$ & TAGAAAGGCTAGGACCAAACCT & 652 & & \\
\hline
\end{tabular}


Table II. mtDNA mutations and corresponding changes of amino acids.

\begin{tabular}{lccccc}
\hline Cell lines & mtDNA NA type & mtDNA mutations & Influenced proteins & Amino acid changes & tRNA \\
\hline H446 & G & G6366A & CO1 & V155I & tRNA-Cys \\
H446/CDDP & & A10086G & ND3 & N10D & I257V \\
& A13105G & ND5 & I189V \\
& A15311G & cytB & T360A \\
& A15824G & cytB & & \\
\hline
\end{tabular}

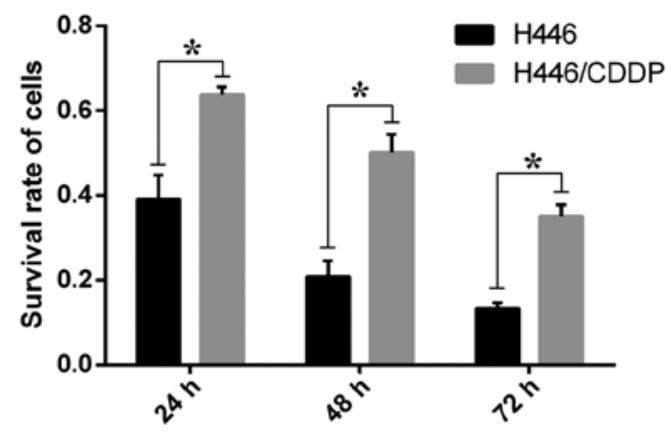

Figure 1. Sensitivity of H446 and H446/CDDP cells to cisplatin was assessed by MTT assay. Cells were treated with $5 \mu \mathrm{g} / \mathrm{ml}$ cisplatin for 24,48 and $72 \mathrm{~h}$. Data are expressed as means $\pm \mathrm{SD}$ of three independent experiments. ${ }^{*} \mathrm{P}<0.01$

lation were detected with DCFH-DA (Beyotime Institute of Biotechnology, China). Briefly, the cells were seeded in laser confocal Petri dishes at a density of $1 \times 10^{4}$ cells/well and incubated for $48 \mathrm{~h}$. Then, $500 \mu \mathrm{l}$ of DCFH-DA working liquid (1:1,500 diluted in RPMI-1640 medium and filtered through a $0.22-\mathrm{mm}$ membrane) was added into each plate. Inverted confocal microscope (FV1000 IX81; Olympus) was used to capture the fluorescence intensity every $5 \mathrm{~min}$ a time since cells were challenged by cisplatin. Finally, fluorescence intensity in the images were digitalized and analyzed by Image-Pro Plus software.

Analysis of mitochondrial membrane potential (MMP). tetrachloro-tetraethylbenzimidazol carbocyanine iodide (JC-1; Beyotime Institute of Biotechnology) is a mitochondrial-specific cationic dye. It is a monomer when the mitochondrial membrane potential is $<120 \mathrm{mV}$ and emits a green light $(540 \mathrm{~nm})$ following excitation by blue light (490 $\mathrm{nm})$. When JC-1 is converted to J-aggregates, a red light $(590 \mathrm{~nm})$ is emitted following excitation by green light (540 nm) (20). In brief, H446 and H446/CDDP cells were plated at a density of $2 \times 10^{5}$ cells/well in a 24 -well plate, and cells were incubated with $5 \mu \mathrm{M} \mathrm{JC}-1$ for $30 \mathrm{~min}$ after stimulation by cisplatin for $24 \mathrm{~h}$ in dark at room temperature. Finally, fluorescence was captured with an inverted fluorescence microscope (DMI6000B; Leica), and changes in the fluorescence intensity ratio between the measurements at wavelengths of $590 \mathrm{~nm}$ (red) and $540 \mathrm{~nm}$ (green) were used to evaluate the MMP.
Western blot analysis. In order to confirm the role of mitochondrial dysfunction in MDR of H446/CDDP cells, activation of the mitochondrial apoptotic pathway was evaluated by western blot analysis. Briefly, H446 and H446/CDDP cells with or without stimulation of cisplatin for $24 \mathrm{~h}$ were collected by centrifugation, and the total protein was extracted by a repeated freezing and thawing method. Equal amounts of protein from each group were separated on $12 \%$ dodecyl sulfate, sodium salt (SDS)-polyacrylamide gel electrophoresis (SDS-PAGE) gels and transferred to a PVDF membrane (Amersham Biosciences, Piscataway, NJ, USA). The membranes were soaked in blocking buffer $\{5 \%$ skimmed milk melted in TBS-T [25 mM Tris (pH 7.6), $138 \mathrm{mM} \mathrm{NaCl}$ and 0.05\% Tween-20]\} for $2 \mathrm{~h}$ and then probed with Bax, cleaved caspase-3, cleaved caspase- 9 and $\beta$-actin (1:1,000-1:5,000; Santa Cruz Biotechnology, Santa Cruz, CA, USA) antibodies overnight at $4^{\circ} \mathrm{C}$. After that, the membranes were further incubated with anti-rabbit IgG HRP-conjugated secondary antibody $(1: 5,000)$. Finally, immune-reactive signals were detected using an ECL detection system (Amersham Pharmacia Biotech).

Statistical analysis. Statistical analysis was performed with SPSS 17.0. Numerical variables are expressed as means \pm SD. Statistical differences between the experimental groups were analyzed by one-way analysis of variance (ANOVA) followed by Dunnett's test. $\mathrm{P}<0.05$ was considered to indicate a statistically significant difference.

\section{Results}

Sensitivity of the H446 and H446/CDDP cells to cisplatin. The survival rate of the control group was taken as 1 , and the results from the different time groups were compared with the control in order to calculate the relative survival rates. The survival rates of the H446 and H446/CDDP cells treated with $5 \mu \mathrm{g} / \mathrm{ml}$ cisplatin were significantly different $(\mathrm{P}<0.01)$ at all three time-points (Fig. 1). The difference in the survival rate between H446 and H446/CDDP cells increased with a decreasing $\mathrm{p}$-value as the time of stimulation was prolonged.

Sequencing results of mtDNA from the $\mathrm{H} 446$ and $H 446 / C D D P$ cells. Amplified mtDNAs of the H446 and H446/CDDP cells were sequenced and compared with rCRS. The mtDNA sequences of the H446 and H446/CDDP cells belonged to haploid type $\mathrm{G}$ (Table II), and several specific base mutations were detected in the mtDNA sequence of both H446 and 

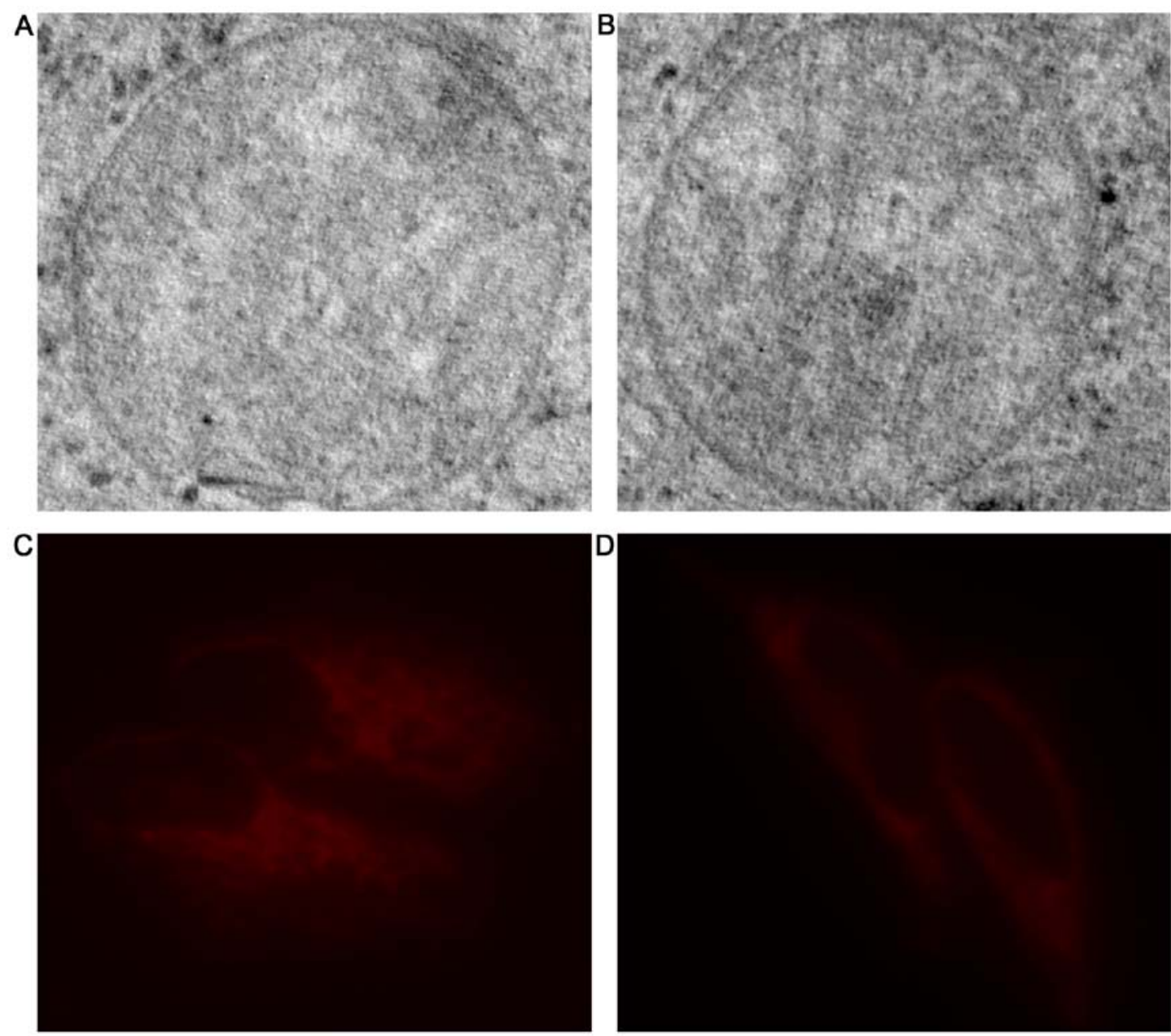

Figure 2. Mitochondrial ultrastructure of H446 (A) and H446/CDDP (B) cells was examined by transmission electron microscopy. Mitochondria in H446 (C) and H446/CDDP (D) cells were stained by mitoTracker probe; fluorescence intensity represents the content of mitochondria.

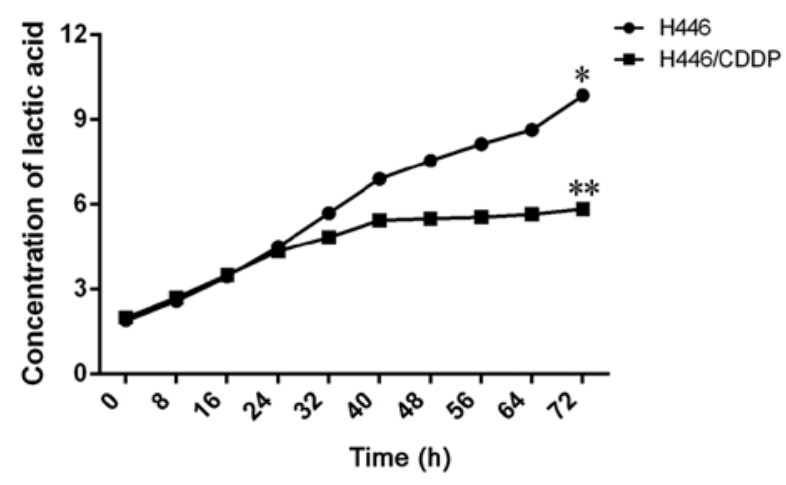

Figure 3. Concentration of lactic acid in the culture supernatant of the H446 and H446/CDDP cells. ${ }^{*} \mathrm{P}$ vs. ${ }^{* *} \mathrm{P}<0.01$.

H446/CDDP cells, which could be related to types of amino acid protein in the mitochondrial respiratory chain and the secondary structure of tRNAs and rRNAs. It was also found that the mtDNA sequence of the H446 cell line was the same as that of the H446/CDDP cells.

Ultrastructure and content of mitochondria in the H446 and H446/CDDP cells. Since there was no difference in the sequences of mtDNA in the H446 and H446/CDDP cells, we explored the structural and functional differences of mitochondria in these two cell lines. Results from electron microscopic observation (Fig. 2A and B) revealed no difference in the ultrastructure of mitochondria in the H446 and H446/CDDP cells. Then, we compared the number of mitochondria in these two cell lines. Mitochondrial staining results revealed that the fluorescence of mitochondria in the $\mathrm{H} 446$ cell line (Fig. 2C) was much stronger than that of the H446/CDDP cells (Fig. 2D).

Lactic acid generation in the H446 and H446/CDDP cells treated with cisplatin. Concentration of lactic acid in the culture supernatant was detected in order to evaluate the metabolic difference in the H446 and H446/CDDP cells. The concentration of lactic acid in the cultural medium from the H446 and H446/CDDP cells increased at about the same speed when stimulated by cisplatin (Fig. 3). However, the generating speed of lactic acid in the H446 cells accelerated $24 \mathrm{~h}$ after stimulation and the concentration of lactic acid in the $\mathrm{H} 446$ cells was extremely higher than that in the H446/CDDP cells $72 \mathrm{~h}$ after of cisplatin treatment $(\mathrm{P}<0.01)$.

ROS generation in the $H 446$ and H446/CDDP cells treated with cisplatin. ROS generation in the $\mathrm{H} 446$ and H446/CDDP 

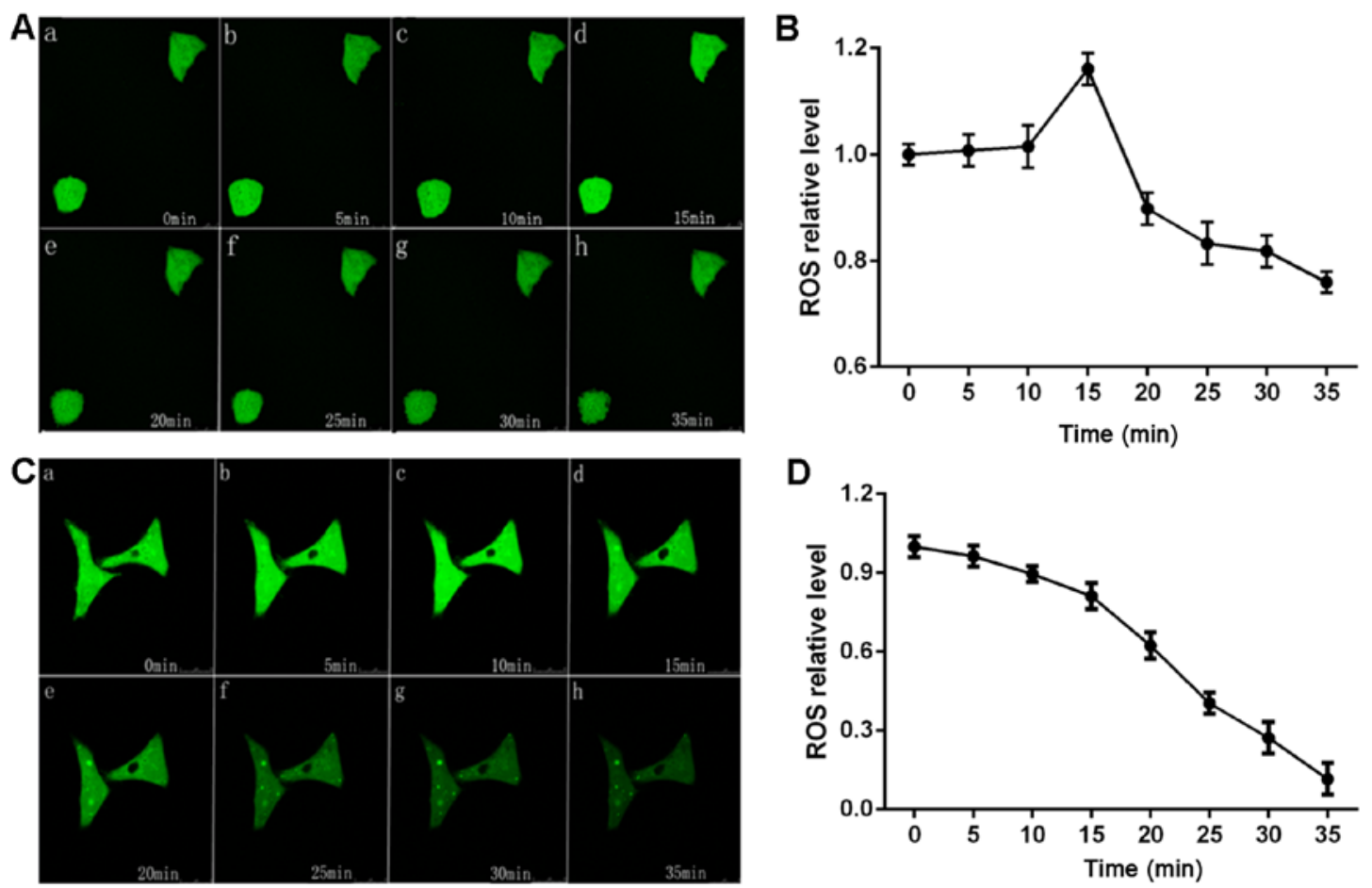

Figure 4. Trends for ROS generation in the (A and B) H446 and (C and D) H446/CDDP cells following treatment with cisplatin.

cells was detected so as to evaluate the ability of these two cell lines to cope with oxidative stress induced by cisplatin. The ROS content in the H446 cells increased and peaked at $15 \mathrm{~min}$ after being stimulated by cisplatin, and then the ROS content slowly decreased (Fig. 4). Meanwhile, H446 cells became shrunken after being stimulated and it was most obvious 35 min after treatment of cisplatin. On the other hand, ROS in the H446/CDDP cells decreased at a relatively stable speed when stimulated with cisplatin and there was no shrinking by this cell line upon stimulation with cisplatin.

Depolarization of the MMP in the H446 and H446/CDDP cells. ROS generation is closely related to depolarization of the MMP which may further lead to cell apoptosis. Depolarization of MMP in H446 and H446/CDDP cells stimulated by cisplatin was evaluated by cationic dye JC-1. The green light became stronger in both the H446 and H446/CDDP cells challenged by cisplatin (Fig. 5). But much stronger green light against red light was detected in the H446 cells compared with that of the H446/CDDP cells, which meant that reduction in the MMP in the H446 cell line was much more obvious than that of the H446/CDDP cells stimulated by $5 \mu \mathrm{g} / \mathrm{ml}$ cisplatin.

Expression of the mitochondrial apoptosis pathway in the H446 and H446/CDDP cells treated with cisplatin. Expression of the mitochondrial apoptosis pathway in the H446 and H446/CDDP cells treated with cisplatin was evaluated by western blotting. Expression of Bax, cleaved caspase- 3 and cleaved caspase- 9 was increased in both the H446 and H446/CDDP cells when challenged by cisplatin (Fig. 6). However, expression levels of these molecules in the H446/CDDP cells were much lower than levels in the H446 cells $(\mathrm{P}<0.05)$.

\section{Discussion}

Mitochondria are intracellular semi-autonomous organelles, which possess a self-owned genome and participate in energy metabolism and homeostasis (21). Human mtDNA is a 16.6-kb double-stranded, circular DNA molecule encoding for 13 complex polypeptides belonging to the mitochondrial respiratory chain, 22 transfer RNAs (tRNAs) and 2 ribosomal RNAs (rRNAs) (21). Since mtDNA is essential for cells, the accumulation of mtDNA mutations and/or reset of mtDNA copy number are believed to affect energy production, cell proliferation as well as cell apoptosis $(21,22)$.

It was first investigated, in the present study, whether mtDNA mutations are associated with the tumorigenesis of SCLC and the MDR phenotype of H446/CDDP cells. mtDNAs from H446 and H446/CDDP cells were amplified, sequenced and compared with rCRS. The results revealed that several spot mutations existed in the mtDNA of SCLC cell lines, which led to changes of amino acids in mtDNA-encoded proteins and base-shifts in rRNA. Astonishingly, the sequences of mtDNA in the H446 cells were the same as that of the MDR cell line H446/CDDP, which meant that mtDNA mutation in sequences was not related to the MDR characteristic of the H446/CDDP cells. Research indicates that both mutations in mtDNA sequences and low mtDNA copy number are closely related to the dysfunction of mitochondria. A reduced mtDNA content could result in impaired mitochondrial respiration and depolarization of the mitochondrial membrane (23). We then detected the mitochondrial content in the H446 and H446/CDDP cells, and found that the fluorescence representing the content of mitochondria in the H446 cells was much stronger than that of H446/CDDP cells. This result was consistent with previous reports indicating that varied mtDNA 

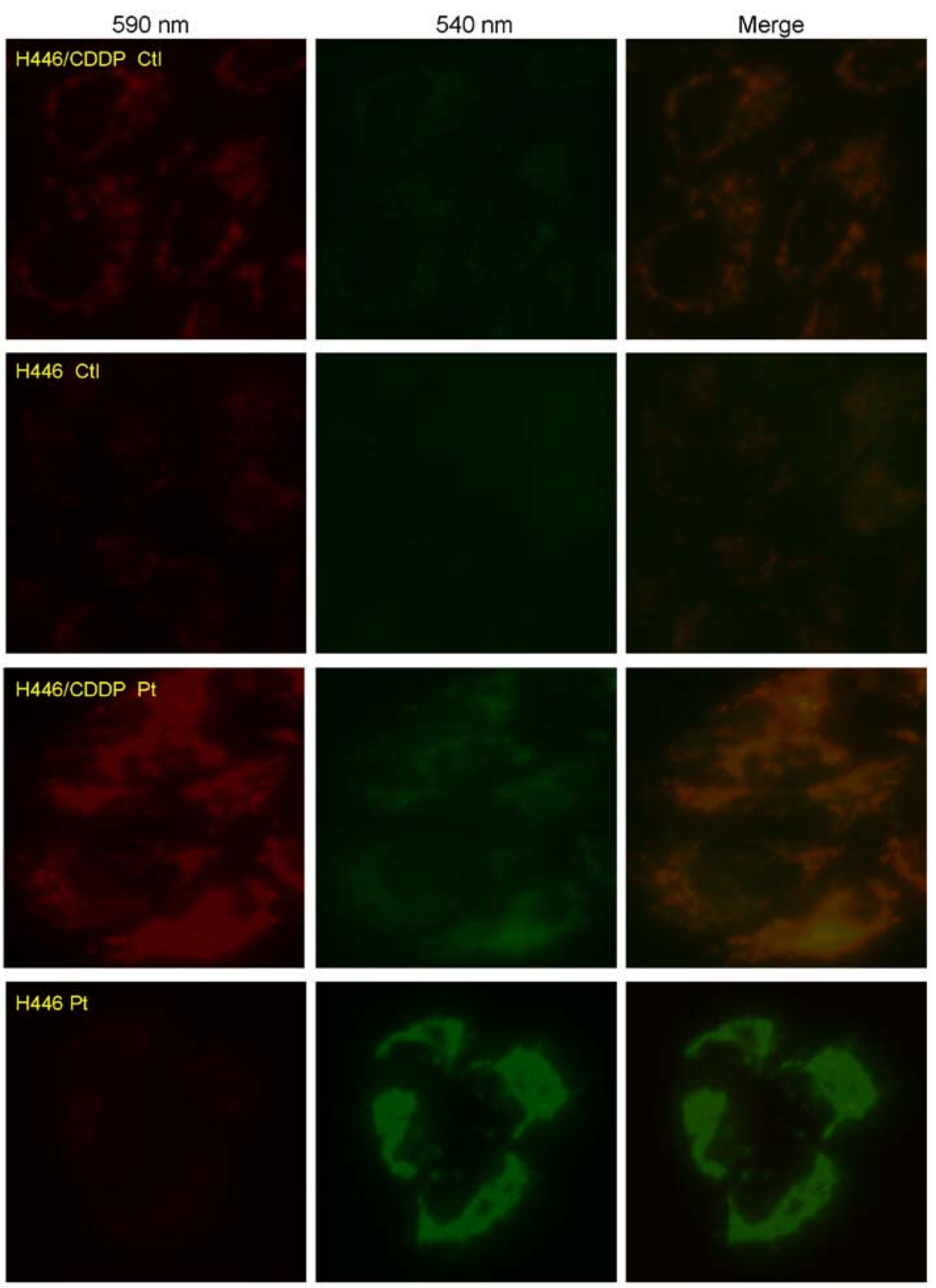

Figure 5. Depolarization of the mitochondrial membrane potential (MMP) in the H446 and H446/CDDP cells was evaluated by JC-1. Changes in the fluorescence intensity ratio between green light $(540 \mathrm{~nm})$ and red light $(590 \mathrm{~nm})$ were used to evaluate MMP.

content markedly increases the risk of lymph node metastasis and high mortality of patients with late-stage tumors (24).

Based on the findings that mutations in mtDNA sequences are responsible for the tumorigenesis of SCLC and varied mitochondrial content in single cells is related to drug sensitivity of H466 and H446/CDDP cells, we wondered how this varied content of mitochondria in the H446 and H446/CDDP cells influenced their sensitivity to cisplatin. As known, mitochondria possess many biological functions, including ATP generation, production of ROS, providing reaction sites for many biological processes and modulating cell apoptosis (25). Evidence also suggests that an energy generation-related 'Warburg effect' may be blamed for the survival benefit of cancer cells $(26,27)$. Lactic acid is an important metabolite of glycolytic activity which could be taken as a marker of anaerobic glycolysis. Generation and secretion of lactic acid from H446 and H446/CDDP cells were evaluated in order to assess their difference in glycolytic activity. Results showed that the H446/CDDP cells generated less lactic acid than that of H446 cells when stimulated by the same dosage of cisplatin, which indicated that a stronger ability of lactic acid removal benefited cancer cell survival.

Then we wondered whether this difference in energy generation was associated with the biological behavior of H446 and H446/CDDP cells. Naito et al (28) reported that mtDNA depletion promotes the chemoresistance of breast cancer cells. In addition, HeLa cells lacking or without mtDNA have been confirmed to become resistant to adriamycin and photodynamics (29). More importantly, Park et al (30) showed that hepatoma cells deprived of mtDNA were less sensitive to hydrogen peroxide and to ROS-inducing agents and further research illustrated that this drug-resistant phenotype was associated with increased expression of antioxidant enzymes manganese superoxide dismutase and glutathione peroxidase. 
A

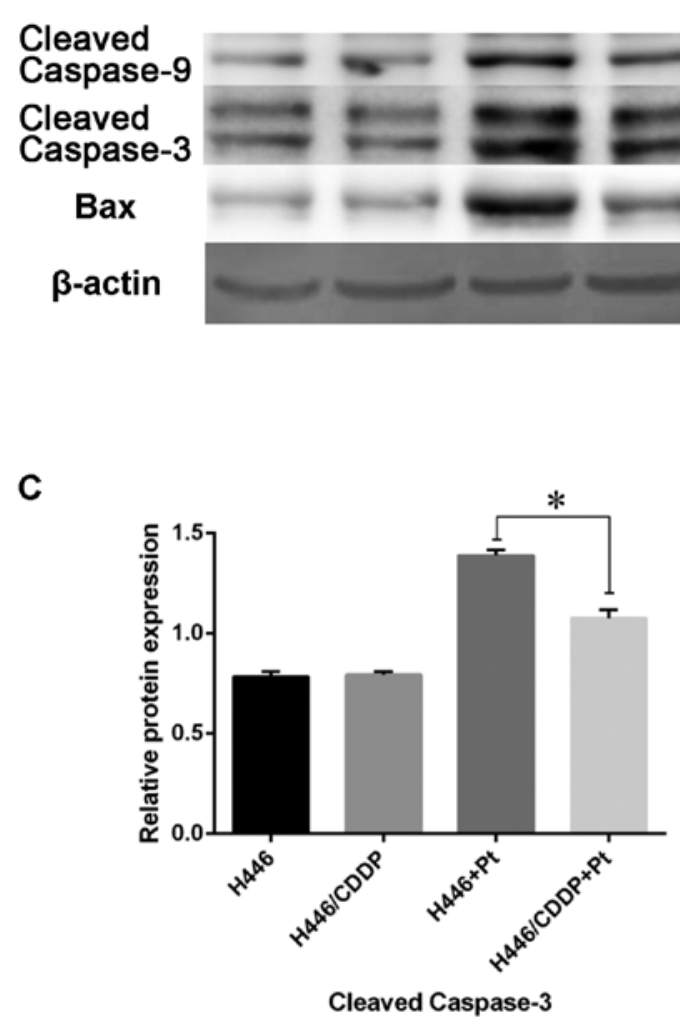

B

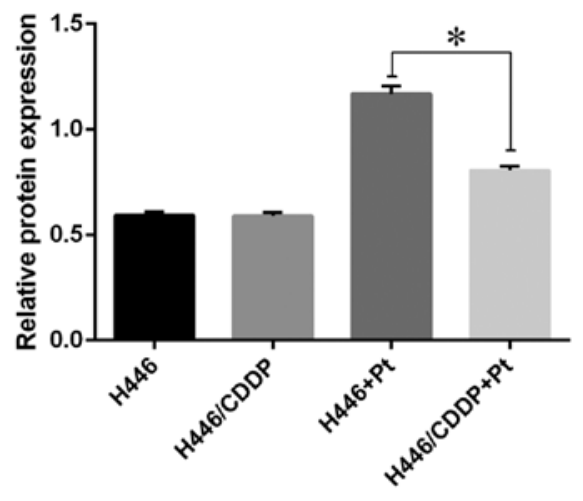

Bax

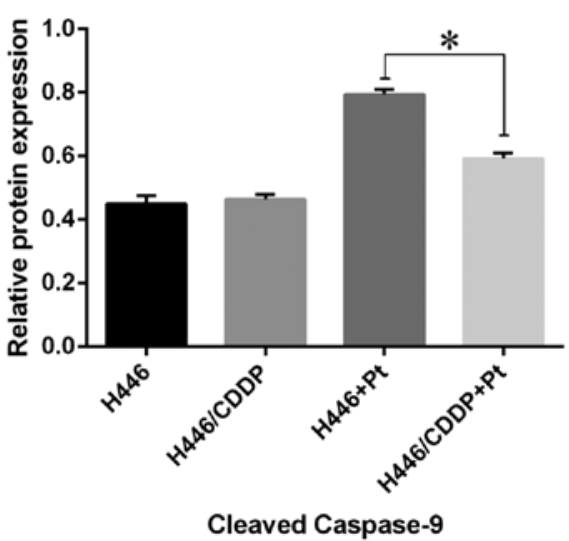

Figure 6. Evaluation of apoptotic regulators in the H446 and H446/CDDP cells stimulated by cisplatin. (A) After protein quantitation, western blot analysis was performed to investigate the contents of Bax, cleaved caspase- 3 and cleaved caspase- 9 . (B-D) The ratios of these proteins vs. $\beta$-actin in three independent experiments were obtained by densitometric scanning using an image analysis system. ${ }^{*} \mathrm{P}<0.05$.

Based on this knowledge, we investigated the production of ROS in H446 and H446/CDDP cells stimulated by cisplatin, and the results showed that more ROS were produced in the H446 cells when challenged by cisplatin compared with that of the H446/CDDP cells. Our findings indicated an upgraded antioxidant capacity in H446/CDDP cells which was also consistent with previous research illustrating that the insensitivity of cancer cells to chemotherapeutic drugs paclitaxel and cisplatin is associated with increased antioxidant expression levels $(31,32)$.

Varied mitochondrial content, difference in lactic acid secretion and ROS generation were confirmed to be related to the discriminative sensitivity of H446 and H446/CDDP cells to cisplatin. While the mechanisms of how those differences participate in the MDR of SCLC remain unknown. As we know, mitochondria are crucial centers of metabolism and ROS production (33). An excess of ROS induces mitochondrial inner membrane permeabilization leading to mitochondrial depolarization, swelling, cytochrome $c$ release and subsequent apoptotic death (34). We further evaluated the activation of the mitochondrial apoptotic pathway in H446 and H446/ CDDP cells challenged by cisplatin. Molecules of the Bcl-2 family were evaluated since they are closely related to the mitochondrial apoptotic pathway, and their abnormal expression leads to loss of $\Delta \Psi \mathrm{m}$, leakage of apoptogenic proteins, activation of caspase-3, and finally cell death (35). Evaluation of the mitochondrial-mediated cell apoptosis pathway showed that cell apoptosis-promoting proteins, Bax, cleaved caspase-3 and cleaved caspase-9, were increased in the H446 cells when compared with the levels in the H446/CDDP cells following stimulation by cisplatin. This indicated that abnormal generation of ROS affected the sensitivity of H446 and H446/CDDP cells to cisplatin probably by interfering with the mitochondrial apoptotic pathway.

In conclusion, mtDNAs from small cell lung cancer cell line H446 and its multi-drug-resistant cell line H446/CDDP were sequenced and compared with rCRS. The results revealed that mtDNA mutations were responsible for the tumorigenesis but not for the MDR capacity of SCLC. On the other hand, a difference in lactic acid and ROS generation confirmed that mitochondrial dysfunction participated in the MDR of SCLC. Finally, evaluation of the ROS-related mitochondrial apoptotic pathway in H446 and H446/CDDP cells challenged by cisplatin explained, at least to some extent, that mitochondrial dysfunction participated in the MDR of SCLC.

\section{Acknowledgements}

The present research was supported by the National Natural Science Foundation of China (no. 81071933).

\section{References}

1. Torre LA, Bray F, Siegel RL, Ferlay J, Lortet-Tieulent J and Jemal A: Global cancer statistics, 2012. CA Cancer J Clin 65: 87-108, 2015.

2. Siegel RL, Miller KD and Jemal A: Cancer statistics, 2015. CA Cancer J Clin 65: 5-29, 2015. 
3. Jun S, Dimyan M, Jones KD and Ladabaum U: Obstipation as a paraneoplastic presentation of small cell lung cancer: Case report and literature review. Neurogastroenterol Motil 17: 16-22, 2005.

4. Sørensen M, Pijls-Johannesma M and Felip E; ESMO Guidelines Working Group: Small-cell lung cancer: ESMO Clinical Practice Guidelines for diagnosis, treatment and follow-up. Ann Oncol 21 (Suppl 5): v120-v125, 2010.

5. Wu X,Zhuang Y and Zhang J: Study on the molecular mechanism of multi-drug resistance in clinical isolates of Mycobacterium tuberculosis. Zhonghua Jie He He Hu Xi Za Zhi 20: 332-335, 1997 (In Chinese).

6. Murakami S: Molecular mechanism of multi-drug resistance. Nihon Rinsho 66: 193-203, 2008 (In Japanese).

7. Warburg $\mathrm{O}$, Wind $\mathrm{F}$ and Negelein E: The metabolism of tumors in the body. J Gen Physiol 8: 519-530, 1927

8. Warburg O: On the origin of cancer cells. Science 3191: 309-314, 1956.

9. Upadhyay M,Samal J,Kandpal M, Singh OV and Vivekanandan P: The Warburg effect: Insights from the past decade. Pharmacol Ther 137: 318-330, 2013.

10. Lee HC, Yin PH, Lin JC, Wu CC, Chen CY, Wu CW, Chi CW, Tam TN and Wei YH: Mitochondrial genome instability and mtDNA depletion in human cancers. Ann NY Acad Sci 1042. 109-122, 2005

11. Compton S, Kim C, Griner NB, Potluri P, Scheffler IE, Sen S, Jerry DJ, Schneider S and Yadava N: Mitochondrial dysfunction impairs tumor suppressor p53 expression/function. J Biol Chem 286: 20297-20312, 2011.

12. Chen JZ, Gokden N, Greene GF, Mukunyadzi P and Kadlubar FF: Extensive somatic mitochondrial mutations in primary prostate cancer using laser capture microdissection. Cancer Res 62 6470-6474, 2002.

13. Heddi A, Faure-Vigny H, Wallace DC and Stepien G: Coordinate expression of nuclear and mitochondrial genes involved in energy production in carcinoma and oncocytoma. Biochim Biophys Acta 1316: 203-209, 1996.

14. Gochhait S, Bhatt A, Sharma S, Singh YP, Gupta P and Bamezai RN: Concomitant presence of mutations in mitochondrial genome and p53 in cancer development - a study in north Indian sporadic breast and esophageal cancer patients. Int J Cancer 123: 2580-2586, 2008.

15. Vivekanandan P, Daniel H, Yeh MM and Torbenson $\mathrm{M}$ : Mitochondrial mutations in hepatocellular carcinomas and fibrolamellar carcinomas. Mod Pathol 23: 790-798, 2010.

16. Larman TC, DePalma SR, Hadjipanayis AG, Protopopov A Zhang J, Gabriel SB, Chin L, Seidman CE, Kucherlapati R and Seidman JG; Cancer Genome Atlas Research Network: Spectrum of somatic mitochondrial mutations in five cancers. Proc Natl Acad Sci USA 109: 14087-14091, 2012.

17. Guha M, Srinivasan S, Ruthel G, Kashina AK, Carstens RP, Mendoza A, Khanna C, Van Winkle T and Avadhani NG: Mitochondrial retrograde signaling induces epithelial-mesenchymal transition and generates breast cancer stem cells. Oncogene 33: 5238-5250, 2014

18. Imanishi H, Hattori K, Wada R, Ishikawa K, Fukuda S, Takenaga K, Nakada K and Hayashi J: Mitochondrial DNA mutations regulate metastasis of human breast cancer cells. PLoS One 6: e23401, 2011.

19. Andrews RM, Kubacka I, Chinnery PF, Lightowlers RN, Turnbull DM and Howell N: Reanalysis and revision of the Cambridge reference sequence for human mitochondrial DNA. Nat Genet 23: 147, 1999.
20. Ovadje P, Chatterjee S, Griffin C, Tran C, Hamm C and Pandey S: Selective induction of apoptosis through activation of caspase- 8 in human leukemia cells (Jurkat) by dandelion root extract. J Ethnopharmacol 133: 86-91, 2011.

21. Wallace DC: Mitochondria and cancer. Nat Rev Cancer 12 685-698, 2012.

22. Wallace DC: A mitochondrial bioenergetic etiology of disease. J Clin Invest 123: 1405-1412, 2013.

23. Guaragnella N, Giannattasio S and Moro L: Mitochondrial dysfunction in cancer chemoresistance. Biochem Pharmacol 92: $62-72,2014$.

24. Zhang G, Qu Y, Dang S, Yang Q, Shi B and Hou P: Variable copy number of mitochondrial DNA (mtDNA) predicts worse prognosis in advanced gastric cancer patients. Diagn Pathol 8: 173,2013

25. Dohi T and Altieri DC: Mitochondrial dynamics of survivin and 'four dimensional' control of tumor cell apoptosis. Cell Cycle 4: 21-23, 2005.

26. Strum SB, Adalsteinsson O, Black RR, Segal D, Peress NL and Waldenfels J: Case report: Sodium dichloroacetate (DCA) inhibition of the 'Warburg effect' in a human cancer patient: Complete response in non-Hodgkin's lymphoma after disease progression with rituximab-CHOP. J Bioenerg Biomembr 45 307-315, 2013.

27. Cui J, Shi M, Xie D, Wei D, Jia Z, Zheng S, Gao Y, Huang S and Xie K: FOXM1 promotes the Warburg effect and pancreatic cancer progression via transactivation of LDHA expression. Clin Cancer Res 20: 2595-2606, 2014.

28. Naito A, Carcel-Trullols J, Xie CH, Evans TT, Mizumachi T and Higuchi M: Induction of acquired resistance to antiestrogen by reversible mitochondrial DNA depletion in breast cancer cell line. Int J Cancer 122: 1506-1511, 2008.

29. Singh KK, Russell J, Sigala B, Zhang Y, Williams J and Keshav KF: Mitochondrial DNA determines the cellular response to cancer therapeutic agents. Oncogene 18: 6641-6646, 1999.

30. Park SY, Chang I, Kim JY, Kang SW, Park SH, Singh K and Lee MS: Resistance of mitochondrial DNA-depleted cells against cell death: Role of mitochondrial superoxide dismutase. J Biol Chem 279: 7512-7520, 2004

31. Hoshida Y, Moriyama M, Otsuka M, Kato N, Taniguchi H, Shiratori Y, Seki N and Omata M: Gene expressions associated with chemosensitivity in human hepatoma cells. Hepatogastroenterology 54: 489-492, 2007.

32. Trachootham D, Alexandre J and Huang P: Targeting cancer cells by ROS-mediated mechanisms: A radical therapeutic approach? Nat Rev Drug Discov 8: 579-591, 2009.

33. Degli Esposti M: Mitochondria in apoptosis: Past, present and future. Biochem Soc Trans 32: 493-495, 2004

34. Salet C, Moreno G, Ricchelli F and Bernardi P: Singlet oxygen produced by photodynamic action causes inactivation of the mitochondrial permeability transition pore. J Biol Chem 272: 21938-21943, 1997.

35. Du W, Hong J, Wang YC, Zhang YJ, Wang P, Su WY, Lin YW, Lu R, Zou WP, Xiong H, et al: Inhibition of JAK2/STAT3 signalling induces colorectal cancer cell apoptosis via mitochondrial pathway. J Cell Mol Med 16: 1878-1888, 2012. 